\title{
A phase I study of preoperative (neoadjuvant) chemotherapy with gemcitabine plus $n a b$-paclitaxel for resectable pancreatic cancer
}

\author{
HIDEHIRO TAJIMA $^{1}$, ISAMU MAKINO ${ }^{1}$, RYOSUKE GABATA ${ }^{1}$, MITSUYOSHI OKAZAKI ${ }^{1}$, \\ YOSHINAO OHBATAKE ${ }^{1}$, HIROYUKI SHIMBASHI ${ }^{1}$, SHINICH NAKANUMA $^{1}$, \\ HIROTO SAITOH $^{2}$, MARI SHIMADA ${ }^{2}$, TAKAHISA YAMAGUCHI ${ }^{2}$, KOICHI OKAMOTO $^{2}$, \\ HIDEKI MORIYAMA $^{2}$, JUN KINOSHITA ${ }^{2}$, KEISHI NAKAMURA ${ }^{2}$, TOMOHARU MIYASHITA ${ }^{1}$, \\ ITASU NINOMIYA ${ }^{2}$, SACHIO FUSHIDA $^{2}$, HIROKO IKEDA $^{3}$ and TETSUO OHTA ${ }^{1}$ \\ Departments of ${ }^{1}$ Hepato-Biliary-Pancreatic Surgery and ${ }^{2}$ Gastroenterological Surgery, \\ Division of Cancer Medicine, Graduate School of Medicine Science, Kanazawa University; ${ }^{3}$ Division \\ of Pathology, Kanazawa University Hospital, Kanazawa, Ishikawa 920-8641, Japan
}

Received June 9, 2020; Accepted September 10, 2020

DOI: $10.3892 / \mathrm{mco} .2020 .2188$

\begin{abstract}
Neoadjuvant chemotherapy (NAC) has become a standard treatment for borderline resectable pancreatic ductal adenocarcinoma (PDAC). The present study examined the maximum tolerated dose of NAC with gemcitabine plus $n a b$-paclitaxel (GnP) in patients with resectable PDAC. Between 2015 and 2019, 39 patients with resectable PDAC were enrolled in the present study. GnP was administered for two 28-day cycles on days 1,8 and 15 . The planned doses for levels 1,2 and 3 were 75,100 and $125 \mathrm{mg} / \mathrm{m}^{2}$, respectively, for $n a b$-paclitaxel and 600,800 and $1,000 \mathrm{mg} / \mathrm{m}^{2}$, respectively, for gemcitabine. Dose-limiting toxicity (neutropenia, anemia, thrombocytopenia and/or liver injury) was observed in $44.4 \%$ of patients treated at dose level 1 (21 patients) and $60.0 \%$ of those treated at dose level 2 (18 patients). Therefore, the maximum tolerated dose was set as level 1. Six patients withdrew from protocol treatment because of non-hematologic adverse events (skin rash, pancreatitis and biliary tract infection). Among the 31 patients with pathologically confirmed PDAC, partial response, stable disease and disease progression were recorded
\end{abstract}

Correspondence to: Dr Hidehiro Tajima, Department of Hepato-Biliary-Pancreatic Surgery, Division of Cancer Medicine, Graduate School of Medicine Science, Kanazawa University, 13-1 Takaramachi, Kanazawa, Ishikawa 920-8641, Japan

E-mail: hidetaji@staff.kanazawa-u.ac.jp

Abbreviations: NAC, neoadjuvant chemotherapy; PDAC, pancreatic ductal adenocarcinoma; GnP, gemcitabine plus nab-paclitaxel; GEM, gemcitabine; PTX, paclitaxel; FOLFIRINOX, 5-fluorouracil, leucovorin, irinotecan, and oxaliplatin; MTD, maximum tolerated dose; DLT, dose-limiting toxicity; DFS, disease-free survival; OS, overall survival; EMT, epithelial-mesenchymal transition

Key words: pancreatic cancer, gemcitabine, nab-paclitaxel, NAC, phase I in $4(12.9 \%), 24(77.4 \%)$ and $3(9.7 \%)$ patients, respectively. NAC significantly reduced tumor size according to computed tomography, and CA19-9 levels and the ${ }^{18} \mathrm{~F}$-fluorodeoxyglucose maximum standardized uptake value were decreased in positron emission tomography. No postoperative complications attributable to NAC were recognized. Among the 27 patients with PDAC who underwent resection, the pathological treatment effect was judged as grades Ia, Ib and II in 21 (77.8\%), $4(14.8 \%)$ and $2(7.4 \%)$ patients, respectively. R0 resection was performed in 24 out of 27 patients (88.9\%). Adjuvant chemotherapy with oral S-1 was administered to 21 out of 27 patients (77.8\%). In conclusion, NAC with GnP was safe and feasible for resectable PDAC at dose level 1. In the future, verification of the long-term results of the present study will be necessary, and a phase II clinical trial is anticipated.

\section{Introduction}

Pancreatic ductal adenocarcinoma (PDAC) is a lethal disease with an extremely low $(<6 \%)$ overall survival (OS) rate $(1,2)$. Recently, 5-fluorouracil, leucovorin, irinotecan, and oxaliplatin (FOLFIRINOX) therapy and gemcitabine (GEM) plus $n a b$-paclitaxel (PTX; GnP) therapy were revealed to improve treatment outcomes among patients with unresectable PDAC $(3,4)$; however, surgery remains the only method for achieving cure or long-term survival. Previously, we attempted radical surgery for patients with localized disease, including wide lymph node dissection and complete removal of the extrapancreatic nerve plexus around the superior mesenteric artery or celiac axis, in an effort to extend survival $(5,6)$. Moreover, in some cases, en bloc resection of the pancreatic head with the superior mesenteric artery was performed (7). However, even in patients who underwent $\mathrm{R} 0$ resection, the 5-year survival rate was poor (7-24\%), and the median survival was approximately 1 year in most series. These unfortunate results indicating that surgery alone is inadequate are likely attributable to early distant metastasis before surgery (8). Therefore, postoperative adjuvant chemotherapy is important (9). Recently, it was 
demonstrated that adjuvant chemotherapy for PDAC with S-1, an oral fluoropyrimidine analog, significantly improved OS and recurrence-free survival after PDAC resection compared with the effects of GEM (10).

An important limitation of adjuvant chemotherapy for PDAC is that a certain proportion of patients cannot receive the designated therapy because of postoperative complications or early disease recurrence (11-13). For this reason, preoperative neoadjuvant chemotherapy (NAC) has attracted attention. The theoretical advantages of NAC include the early treatment of occult metastases, reduction of the risk of tumor seeding during surgery, and improved tolerance compared with postoperative therapy (14). Meanwhile, the potential disadvantages include problems related to biliary drainage during chemotherapy, potential progression to an unresectable stage in patients whose disease does not respond to therapy, and an increasing risk of postoperative complications (14).

Recently, several important studies of preoperative chemotherapy for unresectable or borderline resectable PDAC have been reported (15-18). However, few studies have assessed $\mathrm{NAC}$ in the treatment of resectable PDAC. We previously used NAC for resectable PDAC and found that NAC with GEM-based regimens is useful for pancreatic head cancer with lymph node metastases (19). However, the effectiveness of GnP therapy for patients with resectable PDAC has not been investigated. Therefore, we conducted a phase I study of NAC using GnP for resectable PDAC to determine the maximum tolerated dose (MTD) of each drug.

\section{Materials and methods}

Patient selection. Patients with radiologically proven resectable pancreatic cancer were eligible for this study. Other eligible criteria included an age of 20-79 years; Eastern Cooperative Oncology Group performance status of 0 or 1 ; and adequate renal function (normal serum creatinine and blood urea nitrogen levels), liver function [total bilirubin level, $<2.5 x$ the upper normal limit (UNL) or $<3 x$ the UNL after biliary drainage if the patient had jaundice and serum transaminase (GOT, GPT) levels, and $<2.5 \mathrm{x}$ the UNL or $<3 \mathrm{x}$ the UNL after biliary drainage if the patient had jaundice], bone marrow reserve (white blood cell count, 4,000-12,000 $\mathrm{mm}^{3}$; neutrophil count, $>2,000 \mathrm{~mm}^{3}$; platelet count, $>100,000 \mathrm{~mm}^{3}$; and hemoglobin level, $>9.5 \mathrm{~g} / \mathrm{dl}$ ), and pulmonary function $\left(\mathrm{PaO}_{2},>70 \mathrm{mmHg}\right)$. All enrolled patients were required to have completed or discontinued prior treatment (tumor resection, chemotherapy, immunotherapy, or radiotherapy) at least 4 weeks prior to study enrollment.

The exclusion criteria were as follows: Pulmonary fibrosis or interstitial pneumonia, marked pleural or pericardial effusion or marked peripheral edema, severe heart disease, difficult-to-control diabetes mellitus, active infection, pregnancy or lactation, childbearing age in women who did not use effective contraception, severe drug hypersensitivity, appearance of distant metastases during preoperative chemotherapy, severe neurological impairment or mental disorder, active concomitant malignancy, and other serious medical conditions.

This clinical trial was approved by the Institutional Review Board of Kanazawa University Hospital (no. 5849) and registered with the UMIN Clinical Trials Registry (ID: UMIN000011062).

Study design. This was an open-label, single-center, nonrandomized, dose-escalation phase I study. The primary endpoints of this study were the optimal dose and safety of GnP therapy as preoperative chemotherapy. The secondary endpoints were disease-free survival (DFS), treatment response, and adverse events including postoperative complications. All laboratory tests to assess eligibility were completed within 7 days prior to the start of treatment. $\mathrm{Nab}$-PTX was administered for $60 \mathrm{~min}$ followed by an infusion of GEM for 30 min intravenously on days 1,8 and 15 in two 28-day cycles. Surgery was performed $>14$ days after chemotherapy ended. The dose of each drug in this study was planned as follows: Level $1,75 \mathrm{mg} / \mathrm{m}^{2} /$ day for $n a b$-PTX and $600 \mathrm{mg} / \mathrm{m}^{2} /$ day for GEM; level 2, $100 \mathrm{mg} / \mathrm{m}^{2} /$ day for $n a b$-PTX and $800 \mathrm{mg} / \mathrm{m}^{2} /$ day for GEM; and level $3,125 \mathrm{mg} / \mathrm{m}^{2} /$ day for $n a b$-PTX and $1,000 \mathrm{mg} / \mathrm{m}^{2} /$ day for GEM.

Definition of dose-limiting toxicities (DLTS) and MTD. DLTs were assessed during both treatment cycles. DLT was defined using the National Cancer Institute Common Toxicity Criteria for Adverse Events version 4.0 (20) as one or more of the following effects attributable to the study drug: i) Grade 3/4 neutropenia complicated by fever; ii) grade 4 neutropenia lasting longer than 4 days; iii) grade 4 thrombocytopenia; iv) any other grade $3 / 4$ non-hematologic toxicity excluding anorexia, nausea, and vomiting in the absence of an appropriate antiemetic; and v) delayed recovery from treatment-related toxicity for more than 2 weeks. At least 10 patients were enrolled at each dose level. If DLTs were observed or treatment could not be completed in $<50 \%$ of patients, dose escalation was continued. If DLTs were observed or treatment could not be completed in $>50 \%$ of patients, treatment at that dose was discontinued. The MTD was then set at the preceding dose level.

Assessment of efficacy. Treatment responses were evaluated according to the Response Evaluation Criteria in Solid Tumors (RECIST) version 1.1 (21). Complete response was defined as the disappearance of all clinical evidence of a measurable tumor. Partial response was defined as a $\geq 30 \%$ reduction in the sum of the products of two perpendicular diameters of all measurable lesions compared with the baseline values with no evidence of new lesions. Stable disease (SD) was defined as a $<30 \%$ reduction or $<20 \%$ increase in the sum of the products of two perpendicular diameters of all measurable lesions compared with the baseline values with no evidence of new lesions. Progressive disease (PD) was defined as an increase of $\geq 20 \%$ in the sum of the products of two perpendicular diameters of all measurable lesions compared with the baseline values, the appearance of any new lesion, or deterioration of clinical status consistent with disease progression. To assess objective responses, patients were evaluated after two cycles of preoperative chemotherapy.

Postoperative complications. The global morbidity rate and types of complications were evaluated according to the Clavien-Dindo classification (22). Mortality was defined as any deaths related to surgery. 
Pathological diagnosis. All surgically resected specimens were immediately fixed in $10 \%$ neutral-buffered formaldehyde solution. As described previously, after the specimens had been cut horizontally into 5-mm tissue blocks (23), they were dehydrated and embedded in paraffin. Finally, 5-mm sections were cut and stained with hematoxylin and eosin. Each section was examined by pathologists using light microscopy. The tumors, including the effect of preoperative chemotherapy, were evaluated according to the General Rules for the Clinical and Pathological Study of Pancreatic Cancer proposed by the Japanese Pancreatic Cancer Group (24). The degree of tumor destruction was graded on a scale of 1 to 4 as follows: Grade 1, poor or no response; grade 1a, estimated residual tumor rate $\geq 90 \%$; grade $1 \mathrm{~b}$, residual tumor rate of $50-90 \%$; grade 2 (moderate response), residual tumor rate of $10-50 \%$; grade 3 (marked response), estimated residual tumor rate of $<10 \%$; and grade 4 (complete response), presence of no viable tumor cells.

Statistical analyses. Categorical variables were compared using the Chi-squared test, Student's t-test, and the paired t-test. $\mathrm{P}<0.05$ indicated statistical significance. All analyses were performed using SPSS II statistical software (version 23.0; SPSS, Inc.).

\section{Results}

Patient characteristics. From May 2015 to March 2019, 39 patients (20 males and 19 females) diagnosed with resectable pancreatic cancer were enrolled in this study, as presented in Table I. Preoperative pathological examination was attempted in all cases, but a definitive diagnosis was obtained in only 29 of 39 cases (74.4\%). The median age of patients at the time of study entry was 68 years (range, 46-79). All patients had an Eastern Cooperative Oncology Group performance status of 0 . The tumor location was the pancreatic head in 21 patients and body/tail in 18 patients. Finally, tumor resection was performed for 33 (pancreatoduodenectomy in 17 patients and distal pancreatectomy in 16 patients) of 39 patients entered in this phase I trial (84.6\%). Among the study patients, six (15.4\%) could not undergo tumor resection because of tumor progression.

Toxicity and postoperative complications. As shown in the patient flow chart (Fig. 1), treatment was started at dose level 1 in 21 patients. However, because of non-hematologic adverse events in three patients (two cases of skin rash and one case of biliary tract infection), treatment could not be continued, and these patients withdrew from protocol treatment. The remaining 18 patients were able to complete two treatment cycles at this dose level. During treatment, grade 3 adverse events were observed in eight $(44.4 \%)$ patients (neutropenia, anemia, thrombocytopenia, and/or liver injury), necessitating the skipping of at least one dose (Table II). Afterward, a separate group of 18 patients started treatment at dose level 2; however, three patients withdrew from protocol treatment because of skin rash (two cases) and repeated acute pancreatitis (one case). The remaining 15 patients were able to complete two treatment cycles at this dose level (Fig. 1). During the two cycles, grade 3 or 4 adverse events were observed in 9 of 15 patients $(60.0 \%$; neutropenia, anemia, thrombocytopenia,
Table I. Characteristics of the enrolled patients.

\begin{tabular}{lccc}
\hline Characteristics & Level 1 & Level 2 & Total \\
\hline Patients, $\mathrm{n}$ & 21 & 18 & 39 \\
Sex, $\mathrm{n}$ & & & \\
Male & 13 & 7 & 20 \\
Female & 8 & 11 & 19 \\
Age, years & & & \\
Median & 68.5 & 68.0 & 68.3 \\
Range & $46-78$ & $57-79$ & $46-79$ \\
Location, $\mathrm{n}$ & & & \\
Head & 9 & 12 & 21 \\
Body/tail & 12 & 6 & 18 \\
Surgery, $\mathrm{n}$ & & & \\
PD & 7 & 10 & 17 \\
DP & 85.7 & 83.3 & 84.6 \\
Resection rate, $\%$ & & 5 & 16 \\
\hline
\end{tabular}

PD, pancreatoduodenectomy; DP, distal pancreatectomy.

or liver injury), and administration was skipped for at least one dose (Table II). Therefore, further dose escalation was not performed based on the aforementioned criteria, and the

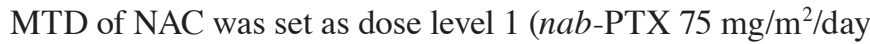
and GEM $600 \mathrm{mg} / \mathrm{m}^{2}$ ) for resectable PDAC. At both dose levels, relatively mild non-hematological toxicities other than skin rash including malaise, nausea, numbness, and anorexia occurred. Hair loss was observed in all patients. Conversely, severe peripheral neuropathies were not observed.

Postoperative complications are listed in Table III. According to the International Study Group on Pancreatic Fistula classification (25), grade B pancreatic fistula, classified as grade IIIa according to the Clavien-Dindo classification, was observed in 4 of 16 patients treated at dose level 1 and 7 of 13 patients treated at dose level 2. Other complications at dose level 1 included intestinal obstruction and diarrhea, and delayed gastric empty, diarrhea, infectious complications (deep surgical site infection), acute appendicitis, and pyogenic spondylitis), and ischemic colitis were observed at dose level 2.

Efficacy. Two patients who underwent resection were determined to not have PDAC (neuroendocrine tumor and PanIN-3), and they were excluded from the evaluation of therapeutic efficacy. Concerning the remaining 31 patients with histologically confirmed PDAC, partial response, $\mathrm{SD}$, and $\mathrm{PD}$ were recorded in $4(12.9 \%), 24(77.4 \%)$, and 3 patients $(9.7 \%)$, respectively, on computed tomography (CT) according to RECIST. Three cases of PD involved distant metastases (liver or peritoneal metastasis), and a patient initially judged to have SD was diagnosed with peritoneal metastasis during surgery. Table IV shows the efficacy data for NAC. Tumor size on CT was significantly decreased from 24.7 to $23.1 \mathrm{~mm}(\mathrm{P}=0.049)$. The CA-19-9 level before treatment was elevated (>37 IU/ml) in 18 of $31(58.1 \%)$ patients. Of these 31 patients, the average CA19-9 level was significantly decreased from 166.1 to $82.5 \mathrm{IU} / \mathrm{ml}$ after 


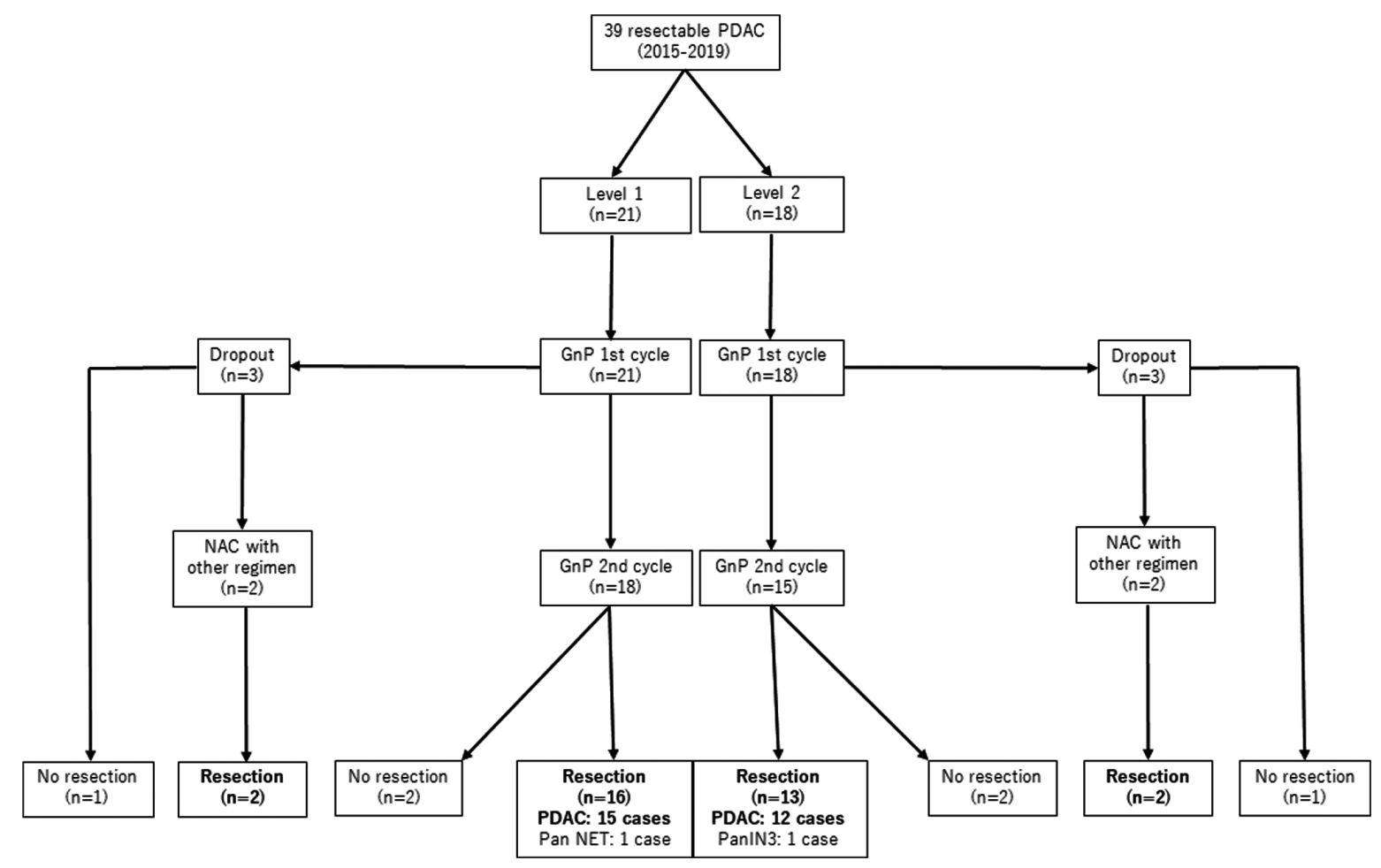

Figure 1. Patient flow chart. A total of 39 patients clinically diagnosed with PDAC were assigned to treatment at dose level 1 (21 patients) or 2 (18 patients). At both levels, three patients withdrew during the first cycle of $\mathrm{GnP}$ therapy, events precluding resection occurred in two patients after the completion of chemotherapy. Finally, 16 patients at dose level 1 and 13 patients at dose level 2 underwent tumor resection. Among the six patients who withdrew from study treatment, NAC was continued with other regimens, and tumor resection was subsequently performed. A total of two patients who underwent resection were determined to have PanNET and PanIN-3, and they were excluded from the evaluation of therapeutic efficacy. GnP, gemcitabine plus nab-paclitaxel; NAC, neoadjuvant chemotherapy; PDAC, pancreatic ductal adenocarcinoma; PanNET, pancreatic neuroendocrine tumor; PanIN, pancreatic intraepithelial neoplasia.

preoperative chemotherapy ( $\mathrm{P}=0.003)$. Significant reductions of CEA and DUPAN-2 levels were not observed after preoperative treatment. Positron emission tomography-CT was performed using ${ }^{18} \mathrm{~F}$-fluorodeoxyglucose (FDG) in all patients, before and after preoperative chemotherapy. A significant decrease in the FDG maximum standardized uptake value from 6.0 to 3.3 was documented after preoperative therapy $(\mathrm{P}=0.001)$.

In the 27 patients who underwent resection as planned, the pathological treatment effect was judged as grade Ia in 21 patients (77.8\%), grade Ib in four patients (14.8\%), and grade II in two patients (7.4\%) patients. There was no case judged as grade 3 or 4. The pathological tumor size was $32.8 \mathrm{~mm}$ (range, 7-65), and lymph node metastasis was detected in 16 of 27 patients (59.3\%). R0 resection was performed in 24 of 27 patients as planned (88.9\%). Although the observation period was short and the data are immature, OS and DFS among these patients were 25.7 and 20.2 months, respectively. Adjuvant chemotherapy with oral S-1 was performed for 21 of 27 patients (77.8\%).

Four patients who withdrew from protocol treatment because of skin rash continued preoperative chemotherapy with other regimens (GEM alone or modified FOLFIRINOX), and $\mathrm{R} 0$ resection was performed for all four patients. Including these patients, $\mathrm{R} 0$ resection was performed in 28 of 37 enrolled patients $(75.7 \%)$.

\section{Discussion}

The National Comprehensive Cancer Network guidelines recommend that NAC with GnP or (modified) FOLFIRINOX should be considered for patients with borderline resectable PDAC (26). Conversely, for resectable PDAC, frontline surgery is recommended for all but high-risk patients. However, patients with pancreatic head cancer in particular require a long postoperative recovery period before adjuvant chemotherapy because of surgical stress. Therefore, preoperative therapy could be expected to reduce the risk of distant metastasis. We introduced NAC for resectable PDAC using GEM plus S-1 in 2007 (14,27). Recently, the effectiveness of NAC with GEM plus S-1 for resectable PDAC was demonstrated in a randomized phase II/III clinical trial (28), and this regimen has become the standard treatment in Japan.

$\mathrm{GnP}$ and FOLFIRINOX are currently in use as NAC for patients with borderline resectable or locally advanced PDAC $(18,29,30)$. Okada et al (31) reported a phase I study of $\mathrm{GnP}$ as NAC for borderline resectable PDAC at doses equivalent to level 3 in the current study ( $n a b$-PTX $125 \mathrm{mg} / \mathrm{m}^{2} /$ day and GEM $1,000 \mathrm{mg} / \mathrm{m}^{2}$ ). The mean frequency of administration in their study was 4.6 of 6 possible doses during two treatment cycles, compared with 5.4 at level 1 and 4.8 at level 2 in our series. From these results, our results are considered valid, but because $<50 \%$ of planned doses were administered at level 2 , we concluded that the MTD was dose level 1 . The most frequent DLT was neutropenia, as described previously $(4,31)$. Whether the dose of the drug should be increased in combination with granulocyte colony-stimulating factor to prevent neutropenia is a future issue. There were no serious adverse events such as sepsis, febrile neutropenia, interstitial pneumonia, and severe peripheral sensory neuropathy in the 
Table II. Grade 3 or 4 adverse events in patients who completed chemotherapy.

\begin{tabular}{lllc}
\hline Adverse event & $\begin{array}{c}\text { Level 1, } \\
\mathrm{n}(\%) \\
(\mathrm{n}=18)\end{array}$ & $\begin{array}{c}\text { Level 2, } \\
\mathrm{n}(\%) \\
(\mathrm{n}=15)\end{array}$ & $\begin{array}{c}\text { Total, } \\
\mathrm{n}(\%) \\
(\mathrm{n}=33)\end{array}$ \\
\hline Anemia & $1(5.6)$ & $0(0.0)$ & $1(3.0)$ \\
Neutropenia & $8(44.4)$ & $9(60.0)$ & $17(51.5)$ \\
Thrombocytopenia & $1(5.6)$ & $1(6.7)$ & $2(5.1)$ \\
Liver dysfunction & $1(5.6)$ & $0(0.0)$ & $1(3.0)$ \\
\hline
\end{tabular}

Table III. Postoperative complications.

\begin{tabular}{lccc}
\hline $\begin{array}{l}\text { Clavien-Dindo's } \\
\text { classification grade }\end{array}$ & $\begin{array}{c}\text { Level 1, } \\
\mathrm{n}(\mathrm{n}=16)\end{array}$ & $\begin{array}{c}\text { Level 2, } \\
\mathrm{n}(\mathrm{n}=13)\end{array}$ & $\begin{array}{c}\text { Total, } \\
\mathrm{n}(\mathrm{n}=29)\end{array}$ \\
\hline Grade I/II & 2 & 1 & 3 \\
$\quad$ Diarrhea & 0 & 1 & 1 \\
$\quad$ Pyogenic spondylitis & & & \\
Grade IIIa & 4 & 7 & 13 \\
$\quad$ ISGPF Grade B & 1 & 0 & 1 \\
Ileus & 0 & 1 & 1 \\
Deep SSI & 0 & 1 & 1 \\
Ischemic colitis & & & \\
Grade IIIb & 0 & 1 & 1 \\
$\quad$ Acute appendicitis & 0 & 0 & 0 \\
Grade IV/V &
\end{tabular}

ISGPF, International Study Group on Pancreatic Fistula; SSI, surgical site infection.

Table IV. Changes in clinical factors after NAC.

\begin{tabular}{lrrr}
\hline Factor & $\begin{array}{r}\text { Before } \\
\text { NAC }\end{array}$ & $\begin{array}{c}\text { After } \\
\text { NAC }\end{array}$ & P-value \\
\hline Tumor size on CT, mm & 24.7 & 23.1 & 0.049 \\
${ }^{18}$ FDG-PET SUVmax & 6.0 & 3.3 & 0.001 \\
CA19-9, U/ml & 166.1 & 82.5 & 0.003 \\
CEA, ng/ml & 3.8 & 4.1 & 0.512 \\
DUPAN-2, U/ml & 649.5 & $2,600.1$ & 0.355 \\
\hline
\end{tabular}

NAC, neoadjuvant chemotherapy; ${ }^{18}$ FDG-PET SUVmax, 18-fluorodeoxyglucose-positron emission tomography maximum standardized uptake value.

current study, probably because of the short-term treatment duration. Skin rash caused by $n a b$-PTX, occurring in four patients (10.0\%), was not a severe adverse event, but this event precluded further protocol treatment. Fortunately, these four patients were able to continue chemotherapy with another regimen (GEM alone or modified FOLFIRINOX therapy) and finally undergo surgical resection.
Postoperative complications attributable to NAC were not recognized. Although infectious complications were observed in patients treated at dose level 2 , these events were considered accidental phenomena. Acute appendicitis developed immediately before discharge, and infectious spondylitis occurred in a patient with steroid-treated autoimmune disease. The most common complication was grade B pancreatic fistula. There was no severe complication classified as grade IV or $\mathrm{V}$ in this study. Two cycles of GnP therapy as NAC are not considered to have safety issues based on these findings.

The most important purposes of preoperative chemotherapy are preventing metastasis from the primary site and treating occult metastasis. Recently, it was reported that epithelial-mesenchymal transition (EMT) of cancer stem cells plays an important role in tumor invasion, metastasis, and chemoradioresistance in PDAC (32). PTX suppresses the EMT of cancer stem cells and activation of cancer-associated fibroblasts (33-36). We previously reported that NAC with GnP reduced the numbers of cancer-associated fibroblasts (37). Therefore, we determined that GnP therapy is most suitable for preoperative chemotherapy theoretically (19).

However, it was recently demonstrated that FOLFIRINOX was associated with longer $\mathrm{OS}$ than $\mathrm{GnP}$ in patients with unresectable locally advanced or metastatic PDAC (38). Moreover, FOLFIRINOX treatment was reported to decrease the frequency of lymph node metastasis $(30,39)$, consistent with our results supporting the effectiveness of NAC in patients with node-positive pancreatic head cancer (19). In addition, in a study limited to NAC for resectable and borderline resectable pancreatic head cancer, the OS of the FOLFIRINOX group was slightly higher than that of the $\mathrm{GnP}$ group, although the difference was not significant because of the limited frequency of treatment in the preoperative period (39).

Recently, many studies on preoperative chemoradiotherapy (CRT) for locally advanced or borderline PDAC have been published and these data demonstrate that CRT improves resectability, R0 resection rate, OS and DFS rate, and reveal a survival advantage compared with NAC cases $(40,41)$. On the other hand, few authors have prospectively evaluated the role of CRT for preoperative treatment for resectable PDAC (42). Theoretically, radiotherapy may have a marked impact on microscopic tumor spread and local disease management. However, it is reported that radiotherapy could induce EMT in cancer cells (43) and increase distant metastasis in patients with locally advanced PDAC (44). In recent years, progression of image diagnosis made possible accurate understand of the tumor spreading (45), for that reason we have adopted optimal resection rather than radiation for local spread of resectable PDAC.

In pancreatic head cancer treatment in particular, postoperative chemotherapy may often be limited by surgical stress, and thus, the concept that more powerful regimens should be used before surgery was also affirmed. Uesaka et al (10) obtained good results for postoperative adjuvant chemotherapy with oral S-1, which has become the standard treatment in Japan, in patients with PDAC. In this study, adjuvant chemotherapy with S-1 was administered to approximately $80 \%$ of patients with PDAC who underwent resection. In our previous study (19), it was revealed that NAC with GEM-based regimens improve prognosis of pancreatic head cancer with lymph node metastases compared with surgery first cases. That study included three 
regimens of preoperative chemotherapy, GEM plus S-1, GEM alone and $\mathrm{GnP}$ therapy. Therefore, we will clarify the long term outcomes of NAC with single regimen of $\mathrm{GnP}$ in the future.

In conclusion, NAC with two cycles of GnP for resectable PDAC was safe and feasible at dose level 1 (nab-PTX $75 \mathrm{mg} / \mathrm{m}^{2} /$ day and GEM $600 \mathrm{mg} / \mathrm{m}^{2} /$ day). In the future, it will be necessary to verify the long-term results of this regimen, and a phase II clinical trial based on the results of this study is anticipated.

\section{Acknowledgements}

Not applicable.

\section{Funding}

No funding was received.

\section{Availability of data and materials}

The datasets used and/or analyzed during the current study are available from the corresponding author on reasonable request.

\section{Authors' contributions}

HT, TM and TO designed the present study. IN and SF performed the statistical and pharmacokinetic analyses. RG, YO, HSh, SN, JK, HSa and MS explained the content of chemotherapy in this clinical trial to patients, obtained their consent, administered chemotherapy and performed pharmacokinetic analyses. TY, KO, HM and KN explained the content of surgery in this clinical trial to patients, obtained their consent, contributed to the clinical trial operation, and were also involved in the pathological diagnosis and statistical analysis. IM, MO and HI created pathological specimens, and made all pathological final diagnoses. All authors read and approved the final manuscript.

\section{Ethics approval and consent to participate}

The clinical trial was approved by the Institutional Review Board of Kanazawa University Hospital (approval no. 5849) and registered with the UMIN Clinical Trials Registry (ID: UMIN000011062), and written informed consent was obtained from each patient at enrolment.

\section{Patient consent for publication}

Not applicable.

\section{Competing interests}

The authors declare that they have no competing interests.

\section{References}

1. Siegel R, Naishadham D and Jemal A: Cancer statistics, 2013 CA Cancer J Clin 63: 11-30, 2013.

2. Cancer Research UK: Pancreatic Cancer Mortality Statistics. Available from: http://www.cancerresearchuk.org/cancerinfo/cancerstats/types/pancreas/mortality/.
3. Conroy T, Desseigne F, Ychou M, Bouché O, Guimbaud R, Bécouarn Y, Adenis A, Raoul JL, Gourgou-Bourgade S, de la Fouchardière $\mathrm{C}$, et al: FOLFIRINOX versus gemcitabine for metastatic pancreatic cancer. N Eng J Med 364: 1817-1825, 2011.

4. Von Hoff DD, Ervin T, Arena FP, Chiorean EG, Infante J, Moore M, Seay T, Tjulandin SA, Ma WW, Saleh MN, et al: Increased survival in pancreatic cancer with nab-paclitaxel plus gemcitabine. N Engl J Med 369: 1691-1703, 2013.

5. Nagakawa T, Kurachi M, Konishi K and Miyazaki I: Translateral retroperitoneal approach in radical surgery for pancreatic carcinoma. Jpn J Surg 12: 229-233, 1982.

6. Nagakawa T, Nagamori M, Futakami F, Tsukioka Y, Kayahara M, Ohta T, Ueno K and Miyazaki I: Result of extensive surgery for pancreatic carcinoma. Cancer 77: 640-645, 1996.

7. Kitagawa H, Tajima H, Nakagawara H, Makino I, Miyashita T, Shoji M, Nakanuma S, Hayashi N, Takamura H, Ohta T and Ohtake H: En bloc vascular resection for the treatment of borderline resectable pancreatic head carcinoma. Mol Clin Oncol 2: 369-374, 2014.

8. Evans DB, Abbruzzese JL and Willett CG: Cancer of the pancreas. In: Cancer: Priciples and Practice of Oncology (6th edition). DeVita VT, Hellman S and Rosenberg SA (eds). Lippincott Williams and Wilkins, Philadelphia, PA, pp1126-1161, 2001.

9. Ottle H, Post S, Neuhaus P, Gellert K, Langrehr J, Ridwelski K, Schramm H, Fahlke J, Zuelke C, Burkart C, et al: Adjuvant chemotherapy with gemcitabine vs observation in patients undergoing curative-intent resection of pancreatic cancer: A randomized controlled trial. JAMA 297: 267-277, 2007.

10. UesakaK,BokuN,Fukutomi A,OkamuraY,KonishiM,MatsumotoI, Kaneoka Y, Shimizu Y, Nakamori S, Sakamoto H, et al: Adjuvant chemotherapy of S-1 versus gemcitabine for resected pancreatic cancer: A phase 3, open-label, randomized, non-inferiority trial (JASPAC 01). Lancet 388: 248-257, 2016.

11. Klinlenbijl JH, Jeekel J, Sahmoud T, van Pel R, Couvreur ML, Veenhof CH, Arnaud JP, Gonzalez DG, de Wit LT, Hennipman A and Wils J: Adjuvant radiotherapy and 5-fluorouracil after curative resection of cancer of the pancreas and periampullary region: Phase III trial of the EORTC gastrointestinal tract cancer cooperative group. Ann Surg 230: 776-784, 1999

12. Spitz FR, Abbruzzese JL, Lee JE, Pisters PW, Lowy AM, Fenoglio CJ, Cleary KR, Janjan NA, Goswitz MS, Rich TA and Evans DB: Preoperative and postoperative chemoradiation strategies in patients treated with pancreaticoduodenectomy for adenocarcinoma of the pancreas. J Clin Oncol 15: 928-937, 1997.

13. Yeo CJ, Abrams RA, Grochow LB, Sohn TA, Ord SE, Hruban RH, Zahurak ML, Dooley WC, Coleman J, Sauter PK, et al: Pancreaticoduodenectomy for pancreatic adenocarcinoma: Postoperative adjuvant chemoradiation improves survival. A prospective, single-institution experience. Ann Surg 225: 621-636, 1997.

14. Tajima H, Ohta T, Kitagawa H, Okamoto K, Sakai S, Makino I, Kinoshita J, Furukawa H, Nakamura K, Hayashi H, et al: Pilot study of neoadjuvant chemotherapy with gemcitabine and oral S-1 for resectable pancreatic cancer. Exp Therap Med 3: 787-792, 2012.

15. Katz MH, Shi Q, Ahmad SA, Herman JM, Marsh Rde W, Collisson E, Schwartz L, Frankel W, Martin R, Conway W, et al: Preoperative modified FOLFIRINOX treatment followed by capecitabine-based chemoradiation for borderline resectable pancreatic cancer: Alliance for clinical trials in oncology Trial A021101. JAMA Surg 151: e161137, 2016.

16. Shubert CR, Bergquist JR, Groeschl RT, Habermann EB, Wilson PM, Truty MJ, Smoot RL, Kendrick ML, Nagorney DM and Farnell MB: Overall survival is increased among stage III pancreatic adenocarcinoma patients receiving neoadjuvant chemotherapy compared to surgery first and adjuvant chemotherapy: An intention to treat analysis of the National cancer database. Surgery 160: 1080-1096, 2016.

17. Nagakawa $Y$, Hosokawa $Y$, Nakayama H, Sahara Y, Takishita $C$, Nakajima T, Hijikata Y, Kasuya K, Katsumata K, Tokuuye K and Tsuchida A: A phase II trial of neoadjuvant chemoradiotherapy with intensity-modulated radiotherapy combined with gemcitabine and S-1 for borderline-resectable pancreatic cancer with arterial involvement. Cancer Chemother Pharmacol 79: 951-957, 2017.

18. Ettrich TJ, Berger AW, Perkhofer L, Daum S, König A, Dickhut A, Wittel U, Wille K, Geissler M, Algül H, et al: Neoadjuvant plus adjuvant or only adjuvant nab-paclitaxel plus gemcitabine for resectable pancreatic cancer-the NEONAX trial (AIO-PAK-0313), a prospective, randomized, controlled, phase II study of the AIO pancreatic cancer group. BMC Cancer 18: 1298, 2018. 
19. Tajima H, Ohta T, Okazaki M, Yamaguchi T, Ohbatake Y, Okamoto K, Nakanuma S, Kinoshita J,Makino I, Nakamura K, etal: Neoadjuvant chemotherapy with gemcitabine-based regimens improves prognosis of node positive resectable pancreatic head cancer. Mol Clinic Oncol 11: 157-166, 2019.

20. Common Terminology Criteria for Adverse Event (CTCAE) Version 4.0. Department of Health and Human Services, National Institutes of Health, National Cancer Institute, May 28, 2009.

21. Eisenhauer EA, Therasse P, Bogaerts J, Schwartz LH, Sargent D, Ford R, Dancey J, Arbuck S, Gwyther S, Mooney M, et al: New response evaluation criteria in solid tumors: Revised RECIST guideline (version 1.1). Eur J Cancer 45: 228-247, 2009.

22. Dino D, Demartines N and Clavian PA: Classification of surgical complications: A new proposal with evaluation in a cohort of 6336 patients and results lf a survey. Ann Surg 240: 205-213, 2004.

23. Makino I, Kitagawa $H$, Ohta $T$, Nakagawara $H$, Tajima $H$, Ohnishi I, Takamura H, Tani T and Kayahara M: Nerve plexus invasion in pancreatic cancer: Spread patterns on histopathologic and embryological analysis. Pancreas 37: 358-365, 2008.

24. Classification of Pancreatic Carcinoma (Fourth English Edition). Japan Pancreas Society, Kanehara \& Co., Ltd., 2017.

25. Bassi C, Marchegiani G, Dervenis C, Sarr M, Abu Hilal M, Adham M, Allen P, Andersson R, Asbun HJ, Besselink MG, et al: The 2016 update of the international study group (ISGPS) definition and grading of postoperative pancreatic fistula: 11 years after. Surgery 161: 584-591, 2017.

26. National Comprehensive Cancer Network: Practice guidelines in oncology for pancreatic adenocarcinoma version 1.2020, 2019.

27. Tajima H, Kitagawa H, Tsukada T, Nakanuma S, Okamoto K Sakai S, Makino I, Furukawa H, Nakamura K, Hayashi H, et al: A phase I study of neoadjuvant chemotherapy with gemcitabine plus oral S-1 for resectable pancreatic cancer. Mol Clinic Oncol 1: 768-772, 2013

28. Motoi F, Kosuge T, Ueno H, Yamaue H, Satoi S, Sho M, Honda G, Matsumoto I, Wada K, Furuse J, et al: Randomized phase II/III trial of neoadjuvant chemotherapy with gemcitabine and S-1 versus upfront surgery for resectable pancreatic cancer (Prep-02/JSAP05). Jpn J Clin Oncol 49: 190-194, 2019.

29. Miyasaka Y, Ohtsuka T, Kimura R, Matsuda R, Mori Y, Nakata K, Kakihara D, Fujimori N, Ohno T, Oda Y and Nakamura M: Neoadjuvant chemotherapy with gemcitabine plus nab-paclitaxel for borderline resectable pancreatic cancer potentially improves survival and facilitates surgery. Ann Surg Oncol 26: 1528-1534, 2019.

30. Macedo FI, Ryon E, Maithel SK, Lee RM, Kooby DA, Fields RC, Hawkins WG, Williams G, Maduekwe U, Kim HJ, et al: Survival outcomes associated with clinical and pathological response following neoadjuvant FOLFIRINOX or gemcitabine/nab-paclitaxel chemotherapy in resected pancreatic cancer. Ann Surg 270: 400-413, 2019.

31. Okada K, Hirono S, Kawai M, Miyazawa M, Shimizu A, Kitahata Y, Ueno M, Hayami S and Yamaue H: Phase I study of Nab-Paclitaxel plus gemcitabine as neoadjuvant therapy for borderline resectable pancreatic cancer. Anticancer Res 37 853-858, 2017.

32. Valle S, Martin-Hijano L, Alcalá S, Alonso-Nocelo M and Sainz B Jr: The ever-evolving concept of the cancer stem cell in pancreatic cancer. Cancers (Basel) 10: 33, 2018.

33. Zhang D, Sun L, Xian W, Liu F, Ling G, Xiao L, Liu Y, Peng Y, Haruna Y and Kanwar YS: Low-dose paclitaxel ameliorates renal fibrosis in rat UUO model by inhibition of TGF-beta/Smad activity. Lab Invest 90: 436-447, 2010.
34. Zhou J, Zhong DW, Wang QW, Miao XY and Xu XD: Paclitaxel ameliorates fibrosis in hepatic stellate cells via inhibition of TGF-beta/Smad activity. World J Gastroenterol 16: 3330-3334, 2010.

35. Choi HS, Savard CE, Choi JW, Kuver R and Lee SP: Paclitaxel interrupts TGF-beta1 signaling between gallbladder epithelial cells and myofibroblasts. J Surg Res 141: 183-191, 2007.

36. Hirose A, Tajima H, Ohta T, Tsukada T, Okamoto K, Nakanuma S, Sakai S, Kinoshita J, Makino I, Furukawa H, et al: Low-dose paclitaxel inhibits the induction of epidermal-mesenchymal transition in the human cholangiocarcinoma CCKS-1 cell line. Oncol Lett 6: 915-920, 2013.

37. Miyashita T, Tajima H, Makino I, Okazaki M, Yamaguchi T, Ohbatake Y, Nakanuma S, Hayashi H, Takamura $H$ Ninomiya I, et al: Neoadjuvant chemotherapy with gemcitabine plus Nab-paclitaxel reduces the number of cancer-associated fibroblasts through depletion of pancreatic stroma. Anticancer Res 38: 337-343, 2018.

38. Chan KKW, Guo H, Cheng S, Beca JM, Redmond-Misner R, Isaranuwatchai W, Qiao L, Earle C, Berry SR, Biagi JJ, et al: Real-world outcomes of FOLFIRINOX vs gemcitabine and nab-paclitaxel in advanced pancreatic cancer: A population-based propensity score-weighted analysis. Cancer Med 9: 160-169, 2020.

39. Dhir M, Zenati MS, Hamad A, Singhi AD, Bahary N, Hogg ME, Zeh HJ III and Zureikat AH: FOLFIRINOX versus gemcitabine/nab-paclitaxel for neoadjuvant treatment of resectable and borderline resectable pancreatic head adenocarcinoma. Ann Surg Oncol 25: 1896-1903, 2018.

40. Polistina F, Di Natale G, Bonciarelli G, Ambfosino G and Frego M: Neoadjuvant strategies for pancreatic cancer. World J Gastroenterol 20: 9374-9383, 2014.

41. Gillen S, Schuster T, Meyer Zum Büschenfelde C, Friess H and Kleeff J: Preoperative/neoadjuvant therapy in pancreatic cancer: A systemic review and meta-analysis of response and resection percentages. PLoS Med 7: e1000267, 2010.

42. Silvestris N, Brunetti O, Vasile E, Cellini F, Cataldo I, Pusceddu V, Cattaneo M, Partelli S, Scartozzi M, Aprile G, et al: Multimodal treatment of resectable pancreatic ductal adenocarcinoma. Crit Rev Oncol Hematol 111: 152-165, 2017.

43. Nakayama A, Ninomiya I, Harada S, Tsukada T, Okamoto K, Nakanuma S, Sakai S, Makino I, Kinoshita J, Hayashi H, et al: Metformin inhibits the radiation-induced invasive phenotype of esophageal squamous cell carcinoma. Int J Oncol 49: 1890-1898, 2016.

44. Van Laethem JL, Hammel P, Mornex F, Azria D, Van Tienhoven G, Vergauwe P, Peeters M, Polus M, Praet M, Mauer M, et al: Adjuvant gemcitabine alone versus gemcitabine-based chemoradiotherapy after curative resection for pancreatic cancer: A randomized EORTC-40013-22012/FFCD-9203/GERCOR phase II study. J Clin Oncol 28: 4450-4456, 2010.

45. Mochizuki K, Gabata T, Kozaka K, Hattori Y, Zen Y, Kitagawa H, Kayahara M, Ohta T and Matsui O: MDCT findings of extrapancreatic nerve plexus invasion by pancreas head carcinoma: Correlation with en bloc pathological specimens and diagnostic accuracy. Eur Radiol 20: 1757-1767, 2010 